\title{
Automatic Scheme for Fused Medical Image Segmentation with Nonsubsampled Contourlet Transform
}

\author{
Ch.Hima Bindu ${ }^{1}$ \\ ${ }^{1}$ Associate.Professor, ECE Department, QIS College of \\ Engineering \& Technology, Ongole, Andhra Pradesh,
}

\author{
Dr.K.Satya Prasad ${ }^{2}$ \\ ${ }^{2}$ Rector \& Professor of ECE Department, JNTU \\ Kakinada, Kakinada, Andhra Pradesh, India.
}

\begin{abstract}
Medical image segmentation has become an essential technique in clinical and research- oriented applications. Because manual segmentation methods are tedious, and semi-automatic segmentation lacks the flexibility, fully-automatic methods have become the preferred type of medical image segmentation. This work proposes a robust fully automatic segmentation scheme based on the modified contouring technique. The entire scheme consists of three stages. In the first stage, the Nonsubsampled Contourlet Transform (NSCT) of image is computed. This is followed by the fusion of coefficients using fusion method. For that fused image local threshold is computed. This is followed by the second stage in which the initial points are determined by computation of global threshold. Finally, in the third stage, searching procedure is started from each initial point to obtain closed-loop contours. The whole process is fully automatic. This avoids the disadvantages of semi-automatic schemes such as manually selecting the initial contours and points.
\end{abstract}

Keywords- Non Sub sampled Contourlet Transform; Image Fusion; Automatic Segmentation.

\section{INTRODUCTION}

In the recent years, medical image segmentation is the main research subject of image processing applications. This performs various types of volumetric and shape comparisons in the middle of different structures. An application of medical image segmentation with respect to segmenting brain is used in research to characterize neurological diseases such as; multiple sclerosis, schizophrenia and Alzheimer's. Accurate brain segmentation provides volume measurements that can detect the onset of degenerative diseases. Medical imaging is separated into structural and functional systems.

Particularly, applications of medical image analysis, video compression, pattern recognition, etc. are explored by using image segmentation schemes. In general, segmentation schemes could be categorized into two principal types: semiautomatic segmentation [1-2] and fully automatic segmentation [7-8]. Semi-automatic segmentation requires selection of initial points for different images.

The active contour scheme proposed by c.xu et al. is based on the initial contour to obtain the correct contour by minimizing local energy function [9-10]. The Falcao et al. developed the LWOF (Live Wire on the Fly) scheme [11] to select the initial point close to the center. The fully automatic segmentation is applied on body parts such as; leg bones [12], brain [13], fingers [14] or ribs [15] to get their contours. The drawback related to this content of images must be known earlier. The robust fully automatic scheme by Yuan et al. is based on modified edge following technique [16], but the computational time of this scheme is increased. Since this segmentation scheme does not need human input to select initial points and threshold values [16-17]. The conventional edge-following technique only analyzes a given current point and next highest point without considering the neighboring points [18]. In this proposed method the fully automatic segmentation is applied on fused multi source medical images to obtain correct contour points by considering all neighboring pixel values of an image.

Image fusion is the process of combining information from two or more images of a scene into a single composite image which is more informative and is more suitable for visual perception or computer processing. The objective in the image fusion is to reduce irresolution and minimize redundancy in the output, while maximizing relevant information particular to an application or task as well.

Given the same set of input images, different fused images may be created depending on the specific application and what is considered as relevant information. There are several benefits in using image fusion: wider spatial and temporal coverage with decreased irresolution, improved reliability and increased robustness of system performance [2].

So far, several fusion algorithms based on multi source medical images have been proposed. The MRI-CT image fusion using edge preserved technique proposed by Xianghi et al, based on multi scale toggle contrast operator [3]. In this paper proposes a new method using NSCT based fusion process for efficient segmentation. The NSCT is a fully shiftinvariant, Multiscale and multi direction expansion that has a fast implementation. The performed computer simulation results showed that the proposed technique was quite efficient. The results are better than the DWT based techniques.

The organization of this paper is as follows, the Section 2 describes the Non Subsampled Contourlet Transform. In Section 3 the generic model of image fusion method. In Section 4 the methodology and the implementation of the proposed process is explained. Section 5 the discussion on the experimental results. In the laconic section the paper is concluded. 


\section{NON SUBSAMPLED CONTOURLET TRANSFORM}

In the foremost contourlet transform down samplers and up samplers are presented in both the laplacian pyramid and the Directional Filter Bank (DFB). Thus, it is not shift-invariant, which causes pseudo-Gibbs phenomena around singularities. NSCT is an improved form of contourlet transform. It is motivated to be employed in some applications, in which redundancy is not a major issue, i.e. image fusion. In contrast with contourlet transform, non-subsampled pyramid structure and non-subsampled directional filter banks are employed in NSCT. The non-subsampled pyramid structure is achieved by using two-channel non subsampled 2-D filter banks. The DFB is achieved by switching off the down samplers/up samplers in each two-channel filter bank in the DFB tree structure and up sampling the filters accordingly. As a result, NSCT is shiftinvariant and leads to better frequency selectivity and regularity than contourlet transform. Figure.1 shows the decomposition framework of contourlet transform and NSCT [20].

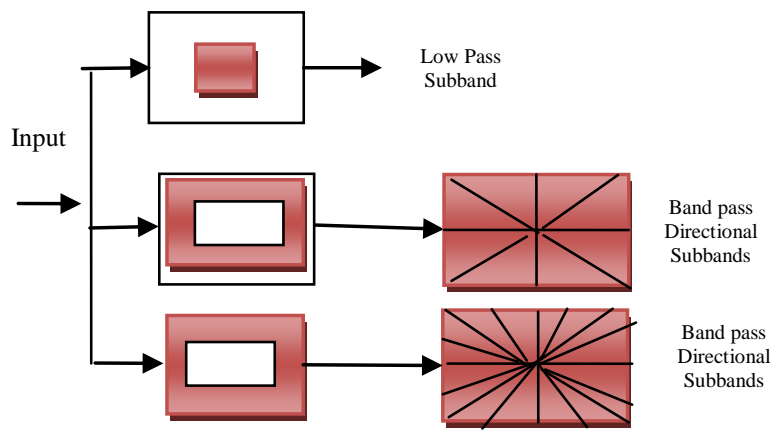

Figure 1: Non Subsampled Contourlet Transform (a) Non Subsampled Filter Bank structure that implements the NSCT.

The NSCT structure consists in a bank of filters that splits the 2-d frequency plane in the subband; these are a non subsampled pyramid structure that ensures the Multiscale property and a non subsampled directional filter bank structure that gives directionality.

\section{NSCT BASED FUSION PROCESS}

In this paper, image decomposition is performed by the NSCT. The NSCT, which are shift-invariant, multiresolution, localization, directionality, and anisotropy, will be more suitable for image fusion and other image processing, i.e. target recognition, object detection, etc. In the fusion process, both neighborhood coefficients and cousin coefficients information are utilized in the salience measure.

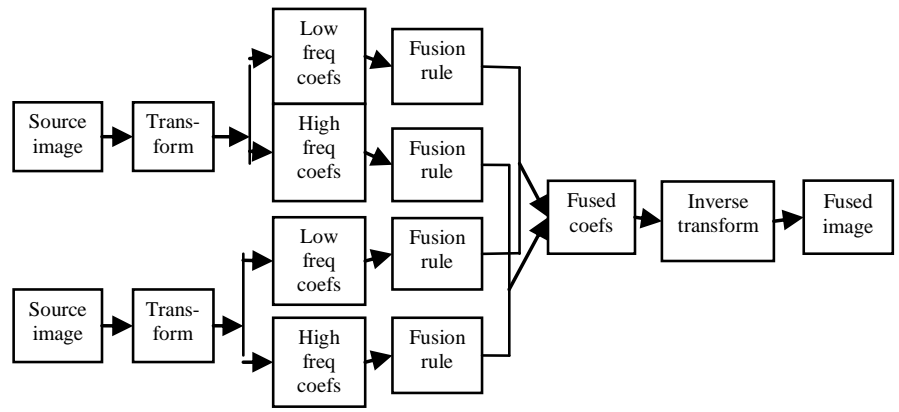

Figure 2: Block diagram of generic model of multi scale image fusion
The Generic model of fusion process is as shown in figure 2 and the rules for fusion of subband coefficients are explained in the following sections:

\section{A. Fusion of Low Frequency subband Coefficients}

The coefficients in the coarsest scale sub band represent the approximation component of the source image. It is a smooth and sub sampled version of the original image. Therefore, most of the source images information is kept in low frequency bands. These bands are fused with average selection rule.The proposed selection principles for the sub band coefficients are finally defined as the average selection rule is:

$$
I_{L}^{F}(i, j)=\left\{\begin{array}{l}
I_{L}^{A}(i, j) \text { if }: I_{L}^{A}(i, j)>I_{L}^{B}(i, j) \\
I_{L}^{B}(i, j) \text { if }: I_{L}^{A}(i, j) \leq I_{L}^{B}(i, j)
\end{array}\right.
$$

\section{B. Fusion of High Frequency subband Coefficients}

High-frequency coefficients contain edge and texture features. In order to make full use of information in the neighborhood and cousin coefficients in the NSCT domain, a salience measure, as a spatial frequency of NSCT coefficients is proposed for the first time. Then spatial frequency measures in the overall activity an image is present. Therefore, we propose a scheme by computing the spatial frequency method in a neighbourhood to select the high frequency coefficients.

The spatial frequency (SF), is originated from the HVS, indicates the overall active level in an image and measure the variation of pixels. For an $\mathrm{M} \times \mathrm{N}$ image I, with gray value I (i, j) at position $(i, j)$ the spatial frequency is defined as:

$$
S F=\sqrt{(R F)^{2}+(C F)^{2}}
$$

Where RF and $\mathrm{CF}$ are the row frequency and column frequency respectively:

$$
\begin{aligned}
R F & =\sqrt{\frac{1}{M N} \sum_{i=1}^{M} \sum_{j=2}^{N}[I(i, j)-I(i, j-1)]^{2}} \\
C F & =\sqrt{\frac{1}{M N} \sum_{i=1}^{N} \sum_{j=2}^{M}[I(i, j)-I(i-1, j)]^{2}}
\end{aligned}
$$

Each image is partitioned into $\mathrm{BxB}$ blocks. The said blocks value varies according to the interest of the user, we consider $8 \times 8$ as a block size to obtain more accurate values. Then, compare the spatial frequencies of two corresponding coefficient values in each blocks of $I_{H I G H}^{A}$ and $I_{H I G H}^{B}$ to construct the new block of fused image $I_{H I G H}^{F}$.

$$
I_{H I G H}^{F}(m, n)=\left\{\begin{array}{cc}
I_{H I G H}^{A}(m, n) & \text { if }: S F_{H I G H}^{A}>S F_{H G H}^{B} \\
I_{H I G H}^{B}(m, n) & \text { if }: S F_{H I G H}^{A}<S F_{H I G H}^{B} \\
\frac{I_{H I G H}^{A}(m, n)+I_{H I G H}^{B}(m, n)}{2} & \text { otherwise }
\end{array}\right.
$$


The resultant fused image is compared with basic DWT method. The results are shown below:

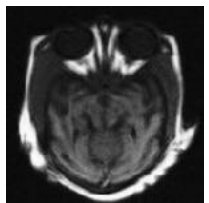

(a)

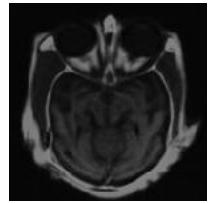

(d)

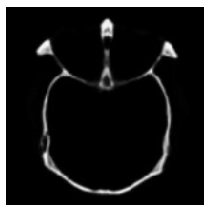

(b)

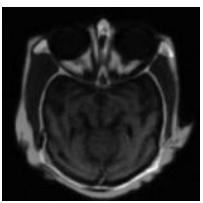

(e)

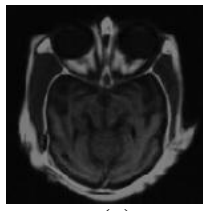

(c)

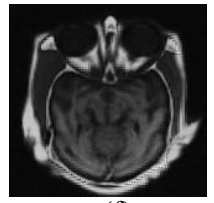

(f)
Figure 3 : Mri-Ct Fusion Results :(A) Source Image A (Mri) (B) Source Image (Ct) (C) Ground Truth Image, (D)-(F) Are Output Fused Images By Dwt With Mean Based Method, Dwt With Spatial Frequency Based Method, Nsct With Proposed Method.

\section{PROPOSED METHOD}

The entire procedure consists of three stages. The final output is automatic segmented image. The fully automatic segmentation process steps are as follows [19]:

Step1: Read the two different source medical images to be fused (MRI/CT Scan images).

Step2: Perform image fusion on two different images with NSCT fusion process.

Step3: Determine the local threshold

The fused image is partitioned into B X B blocks. The maximum of the difference between the right and left neighboring points in direction of $\mathrm{d}$ are defined as $\boldsymbol{E}_{m, n}(i, j)$ in eq. (6).

$$
E_{m, n}(i, j)=\operatorname{MAX}\left(\left|I^{F}\left(r_{m, n}^{d}(i, j)\right)-I^{F}\left(l_{m, n}^{d}(i, j)\right)\right|\right)
$$

Where $\mathrm{d}=0$ to 3 .

Where $r_{m, n}^{d}(i, j)$ and $l_{m, n}^{d}(i, j)$ are the right and the left neighboring points of $(i, j)$ in direction of $d$ respectively.

$$
\begin{gathered}
T_{g m, n}=\max \left(E_{m, n}(i, j)\right) \\
T_{g}=\frac{\min \left(T_{g m, n}\right)+\max \left(T_{g m, n}\right)}{2}
\end{gathered}
$$

The coordinates of each block in an image frame are $(m, n)$ both $\mathrm{m}$ and $\mathrm{n}$ range from 0 to $\mathrm{B}-1$. The coordinates in each block are $(\mathrm{x}, \mathrm{y})$, where $\mathrm{x}$ ranges from 0 to $(\mathrm{M} / \mathrm{B})-1$ and $\mathrm{y}$ ranges from 0 to $(\mathrm{N} / \mathrm{B})-1 . \mathrm{M}$ and $\mathrm{N}$ represent the width and the height of the image, respectively.

Step 4: Finding the global threshold for entire image using equation (12) after $\mathrm{T}$ is computed, Here the $\mathrm{m}$ ranges from 0 to $\mathrm{M}-1$ and $\mathrm{n}$ ranges from 0 to $\mathrm{N}$-1.This indicates the whole image to compute the relation between all possible neighboring directions is considered. i.e., $E(i, j)$
Step 5: Obtain the closed loop contours if $E(i, j)$ is greater than $\mathrm{T}$, then the pixel value is set at 1 otherwise 0 . This gives segmented output.

\section{EXPERIMENTAL RESULTS AND DISCUSSION}

The most essential task of the segmentation process is the discrimination of each spot's foreground from its background. The experiment is performed on different MRI \& CT scan images of body. The same experiment also performed on individual medical images with sub band coefficient fusion method. The all segmented results give almost closed contours and gives detailed analysis. The various segmented results are shown below which are compared with basic DWT method:

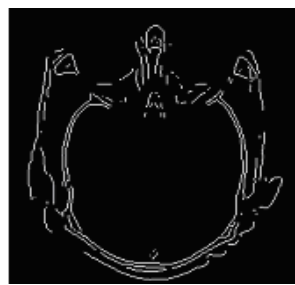

(a)

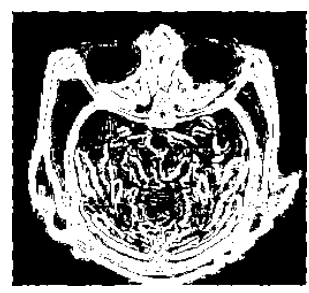

(c)

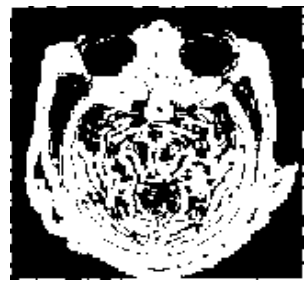

(b)

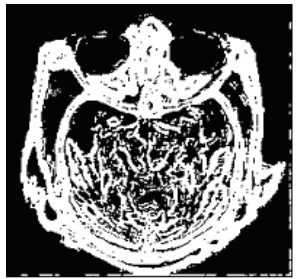

(d)
Fig 4: Edge detected outputs of MRI-CT (a) Conventional edge method, (b)(d) edge detection with Automatic Segmentation approach (b) DWT (fusion with mean values) (c) DWT (fusion with spatial frequency technique) (d) NSCT method (proposed method).

\section{A. Mutual Information (MI)}

It is a metric defined as the MI between each source image and fused image. Considering two source images $\mathrm{X} \& \mathrm{Y}$ and fused image $\mathrm{Z}$.

$$
\begin{aligned}
& \boldsymbol{I}_{Z, X}(Z, X)=\sum \boldsymbol{P}_{Z, X}(Z, X) \log \frac{\boldsymbol{P}_{Z, X}(Z, X)}{\boldsymbol{P}_{Z}(Z) \boldsymbol{P}_{X}(x)} \\
& \boldsymbol{I}_{Z, Y}(Z, Y)=\sum \boldsymbol{P}_{Z, Y}(Z, Y) \log \frac{\boldsymbol{P}_{Z, Y}(Z, Y)}{\boldsymbol{P}_{Z}(z) P_{Y}(y)}
\end{aligned}
$$

Where $\mathrm{P}_{X}, \mathrm{P}_{\mathrm{Y}}$ and $\mathrm{P}_{\mathrm{Z}}$ are probability density function in the images $\mathrm{X}, \mathrm{Y}$ and $\mathrm{Z}$ respectively. $\mathrm{P}_{\mathrm{Z}, \mathrm{X}}$ and $\mathrm{P}_{\mathrm{Z}, \mathrm{Y}}$ are joint probability functions. Thus the image fusion performance measure can be defined as

$$
M I=I_{Z, X}(Z, X)+I_{Z, Y}(Z, Y)
$$

The larger the value of mutual information the better is the fusion result.

\section{B. Peak Signal to Noise Ratio}

The more subjective qualitative measurement of distortion is the Peak Signal -to-Noise Ratio (PSNR).It uses a constant 
value in which to compare the against instead of a fluctuating signal as in SNR.

$$
P S N R=10 \log \left|\frac{255^{2}}{\frac{1}{M N} \sum \sum(G(m, n)-Z(m, n))^{2}}\right|
$$

TABLE 1: EVALUATION OF FUSED IMAGES

\begin{tabular}{ccc}
\hline Algorithm & $\begin{array}{c}\text { PSNR } \\
(\mathbf{d b})\end{array}$ & $\begin{array}{c}\text { MI } \\
\text { (bits) }\end{array}$ \\
\hline DWT & 15.739 & 1.11 \\
$\begin{array}{c}\text { (fusion with mean values) } \\
\text { DWT }\end{array}$ & 24.59 & 2.458 \\
$\begin{array}{c}\text { (fusion with Spatial frequency } \\
\text { technique) } \\
\text { NSCT Method } \\
\text { (proposed method) }\end{array}$ & $\mathbf{2 5 . 0 7 6}$ & $\mathbf{2 . 8 0 1}$ \\
& &
\end{tabular}

The template is used to format your paper and style the text. All margins, column widths, line spaces, and text fonts are prescribed; please do not alter them. You may note peculiarities.

For example, the head margin in this template measures proportionately more than is customary. This measurement and others are deliberate, using specifications that anticipate your paper as one part of the entire proceedings, and not as an independent document. Please do not revise any of the current designations.

\section{CONCLUSION}

In this paper, the improved approach of a fully automatic segmented method without any manual interaction was proposed. This method integrates multi scale image segmentation and a statistical fusion scheme. With this proposed method computing and analyzing the characteristics of the left and right neighboring points of the next estimated contour points has the ability to overcome noise interference.

One of the main importance of proposed scheme does not need human input to select initial points and threshold values. This method can easily be extended to locate the closed loop contours of general images with small change on setting the threshold value. This fully automatic segmentation scheme as proposed herein can accurately and repeatedly segment multiple objects for various image and video applications.

\section{REFERENCES}

[1] Y. B. Chen, T.-C. Chen, Semi-Automatic image segmentation using dynamic direction prediction, Proc. Of IEEE ICASSP 2002, Vol. 4, May 2002, pp.3369-3372.

[2] K.Karsch, Q.He, Y.Duan, A fast, semi-automatic brain structure algorithm for magnetic resonance imaging, Proc. Of IEEE BIBM 2009, November 2009, pp.297-302.

[3] Sabalan Daneshvar, Hassan Ghassemian, MRI and PET Image Fusion by Combining HIS and retina - inspired Models, Information Fusion 11, 2010, pp.114-123.

[4] Guest Editorial, Image Fusion: Advances in the State Of The Art, Information Fusion 8, 2007, pp.114-118.

[5] Xiangzhi Bai, Fugen Zhou, Bindang Xue, Edge Preserved Image Fusion Based on Multiscale Toggle Contrast Operator, Image And Vision Computing 29, 2011, pp. 829-839.
[6] Yuhui Liu, Jinzhu Yang, Jinshan Sun, PET/CT Medical Image Fusion Algorithm Based On Multiwavelet Transform, $2^{\text {nd }}$ International conference on advanced computer and control, 2010, pp.264-268.

[7] Y. B. Chen, T.-C. Chen, Semi-Automatic image segmentation using dynamic direction prediction, Proc. Of IEEE ICASSP 2002, Vol. 4, May 2002, pp.3369-3372.

[8] K.Karsch, Q.He, Y.Duan, A fast, semi-automatic brain structure algorithm for magnetic resonance imaging, Proc. Of IEEE BIBM 2009, November 2009, pp.297-302.

[9] Y.B. Chen, O.T.C. Chen, Image segmentation method using thresholds automatically determined from picture contents, EURASIP j.Image Video Process, 2009 (15).

[10] Y.B.Chen,O.T.C.Chen, High accuracy moving object extraction using background subtraction, ICIC Express Lett., December 2009 , pp.649652.

[11] C.Xu, J.L. Prince, Snakes, Shapes and Gradient Vector Flow, IEEE Trans. Image Process, 7 March 1998, pp.321-331.

[12] W.N.Lie, C.H.Chaung, Fat and accurate snake model for object contour detection, Electron Lett. 37(10), May 2010 (624-626).

[13] A.X.Falco, J.K.Udupa, F.K. Miyazawa, An ultra fast user steered image segmentation paradigm: live wire on the fly, IEEE Trans. Med. Image. 19(1), January 2000, pp.56-62.

[14] Y.B.Chen, Oscal T-C Chen, H.T.Chang, J.T. Chein, An automatic medical assistance diagnosis system applicable on X-ray images. Proc. Of IEEE MWCAS August 2001, pp.910-914.

[15] G.B.A boutanos, J.Nikanne, N.Watkins, B.M. Dawant, Model creation and deformation for the automatic segmentation of the brain in MRI images, IEEE Trans. Biomed. Eng. 46(11),

[16] D.J.Michal, .C. Nelson, HANDX: A model based system for automatic segmentation of bones from digital hard radiog raphs, IEEE Trans. Med. Imag November 1999 (1346-1356) 8(1), March 1989, pp. 64-69.

[17] Z.Yue, A. Goshtasby, L.V.Ackerman, Automatic detection of rib borders in chest radiographs, IEEE Trans. Med. Image 14(3), September 1995, pp.525-536.

[18] Yuan Been Chen, A robust fully automatic scheme for general image segmentation, Digital Signal Processing (21), 2011, pp.87-99.

[19] Ch. Hima Bindu, Dr.K.Satya Prasad, A Fully Automatic Scheme for Medical Image Segmentation With Wavelet Based Image Fusion, Proceedings of the 2011 International Conference on Image Processing, Computer Vision \& Pattern Recognition, July 2011, pp.230-235.

[20] Arthur L.da Cuncha,JIANPING Zhou, "The Non subsampled Contourlet transform: Theory, Design and Applications." IEEE Transactions on Image Processing, Vol 15, Oct2006.

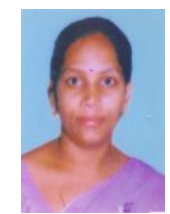

\section{AUTHORS PROFILE}

Ch.Hima bindu is currently working as Associate Professor in ECE Department, QIS College of Engineering \& Technology, ONGOLE, and Andhra Pradesh, India. She is working towards her Ph.D. at JNTUK, Kakinada, India. She received her M.Tech. from the same institute. She has ten years of experience of teaching undergraduate students and post graduate students. She has published 10 research papers in International journals and more than 8 research papers in National \& International Conferences. Her research interests are in the areas of image Segmentation, image Feature Extraction and Signal Processing.

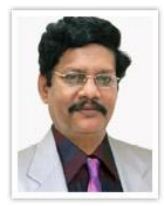

Dr.K.Satya Prasad is currently Rector and Professor in ECE Department, JNTUK, Kakinada, India. He received his Ph.D. from IIT, Madras. He has more than 32 years of experience in teaching and 25 years of R \& D. He is an expert in Digital Signal Processing. He guided 10 PhD's and guiding $10 \mathrm{PhD}$ scholars. He authored Electronic Devices and Circuits, Network Analysis and Signal \& Systems text books. He held different positions in his carrier like Head of the Department, Vice Principal, Principal for JNTU Engg College and Director of Evaluation \& presently the Rector of JNTUK.. He published more than 100 technical papers in national and International journals and conferences. The area of interest includes Digital Signal Processing, Image Processing, Communications etc. 